\title{
Alpha-Synuclein Dopaminylation Presented in Plasma of Both Healthy Subjects and Parkinson's Disease Patients
}

\author{
Huiyuan Zhao, Shuai Huang, Sivakumar Palanisamy, Cui Wang, Gregor Rainer, \\ and Xiaozhe Zhang*
}

Purpose: Alpha-synuclein ( $\alpha$-syn) dopaminylation can lead to the death of dopaminergic neurons in the brain and is a risk factor of Parkinson's disease (PD). This study aims to examine whether such a posttranslational modification (PTM) is presented in human blood plasma.

Experimental Design: In vitro reaction simulation between $\alpha$-syn and dopamine (DA) is conducted to study the biochemical mechanism. Then $\alpha$-syn from human blood plasma samples is detected by using immunoprecipitationmass spectrometry (IP-MS). Lastly the levels of endogenous $\alpha$-syn and $\alpha$-syn dopaminylation in 88 blood plasma samples from patients with PD, major depressive disorder (MDD), and healthy control (HC) are compared. Results: DA modifies $\alpha$-syn with the addition of dopamine-quinone (DAQ) into lysine sites of $\alpha$-syn in vitro and the addition of DAQ and 3,4-dihydroxyphenylacetaldehyde (DOPAL) in plasma samples. The unmodified $\alpha$-syn between the PD and HC groups showed similar levels. The levels of two peptides, one with lysine $34\left({ }^{34} \mathrm{~K}\right)$ DAQ modification and the other with lysine $23\left({ }^{23} \mathrm{~K}\right)$ ubiquitination, are significantly higher in PD and MDD compared with HC.

Conclusions and Clinical Relevance: Thus, $\alpha$-syn dopaminylation is measurable and might be used to indicatethe presence and progression of neurological disorders.

\section{Introduction}

Alpha-synuclein ( $\alpha$-syn) is a 140 -amino acid protein $(14.4 \mathrm{kDa}$; $\mathrm{pKa}$ of 4.7$)$ that is characterized by three regions. ${ }^{[1]}$ An

\footnotetext{
H. Zhao, Prof. G. Rainer

Section of Medicine

University of Fribourg

Fribourg $\mathrm{CH} 1700$, Switzerland

H. Zhao, S. Huang, Dr. S. Palanisamy, Prof. X. Zhang

Division of Biological Technology, Dalian Institute of Chemical

Physics

Chinese Academy of Science

Dalian, China 116023

E-mail: zhangxz@dicp.ac.cn

C. Wang

Department of Neurology

Dalian Central Hospital

Dalian, China 116033
}

amphipathic lysine-rich amino terminus (1-64) includes the mutation sites of familial Parkinson's disease (PD) (A30P, $\mathrm{E} 46 \mathrm{~K}, \mathrm{~A} 53 \mathrm{~T})$. The second region is a nonamyloid- $\beta$ component of amyloid plaques (NAC region, 65-90) which is indispensable for $\alpha$-syn aggregation. ${ }^{[2-5]}$ The third region is an acidic carboxyl-terminal tail (91-140) that has been implicated in regulating $\alpha$-syn nuclear localization and interactions with metals, small molecules and proteins. ${ }^{[6]} \alpha$-Syn is a cytosolic protein that is abundantly expressed in the central nervous system (CNS). It is released in small amounts from nerve cells via unconventional exocytosis. ${ }^{[7]} \alpha$-Syn can adopt various conformations from monomeric to fibrillar, from unfolded in solution to amyloid structure in the fibrils that compose the Lewy body. ${ }^{[1,8,9]} \mathrm{PD}$ is characterized by a progressive degeneration of dopaminergic neurons in the substantia nigra pars compacta, which eventually leads to a malfunction of basal ganglia circuits producing motor and cognitive symptoms of PD. ${ }^{[10]}$ Lewy body formation often co-occurs with PD and involves aggregation of $\alpha$-syn. In PD and other synucleinopathies, the structure

and function of $\alpha$-syn, including aggregation, is influenced by multiple factors, which include gene mutations, posttranslational modifications (PTMs), abnormal physical conditions, oxidative pressure, and so on.

DA has been implicated as an important risk factor for $\alpha$-syn modification and aggregation. ${ }^{[11-13]}$ DA induces oligomerization of $\alpha$-syn in vitro ${ }^{[13]}$ and in vivo, ${ }^{[14]}$ leading to the selective loss of dopaminergic neurons in PD. This relationship between DA and $\alpha$-syn has been dubbed "tango". ${ }^{[1,15]}$ On one hand, genetic mutation and the abnormal PTMs can induce oligomerization of $\alpha$-syn, which can impair dopaminergic neurons, in turn impaired neurotransmitter storage arising from mutations in $\alpha$-syn could lead to cytoplasmic accumulation of DA. ${ }^{[10]}$ On the other hand, the increased level of cytoplasmic DA might result in DA oxidation and the generation of reactive oxygen species $(\mathrm{ROS})^{[10]}$ that could modify the structure of $\alpha$-syn, eventually leading to $\alpha$-syn aggregation, Lewy body formation, and death of neurons.

In vitro evidence suggests that DA, ${ }^{[16,17]}$ DA derivatives, ${ }^{[18-20]}$ and DA analogues ${ }^{[21,22]}$ can contribute to the modification of the structure of $\alpha$-syn. This process involves oxidation of amino 
acids, including residues of methionine, ${ }^{[23]}$ tyrosine, ${ }^{[24]}$ and di-tyrosine. ${ }^{[25]}$ For example, 3,4-Dihydroxyphenylacetaldehyde (DOPAL, an in vivo derivative of DA) interacts with $\alpha$-syn by the addition of two DOPAL molecules to the lysine side chain amine through their aldehyde moieties to forma pyrrole ring. ${ }^{[18]}$ Furthermore, DOPAL also oxidises an $\alpha$-syn methionine to methionine-sulfoxide in vitro. ${ }^{[20]}$ In addition, the 125YEMPS129 motif has also been identified as an interaction site for DA and $\alpha$-syn. ${ }^{[26-28]}$ Chemical binding between DA and $\alpha$-syn likely involves a covalent linkage via hydrogen bonding or hydrophobic interactions. ${ }^{[29]}$ This interaction is strong, and is not dissociated by $6 \mathrm{~m}$ guanidine hydrochloride. ${ }^{[30]}$ Meanwhile, a non-covalent interaction between DA and $\alpha$-syn has been identified ${ }^{[22,31]}$ by molecular dynamics simulations. ${ }^{[21]}$ Taken together, many studies have investigated modification of $\alpha$-syn by DA in vitro. However, few studies have targeted modification of $\alpha$ syn in biological samples that reflects endogenous physiological processes.

DA modification of $\alpha$-syn represents one kind of PTM. In addition, conventional $\alpha$-syn PTMs have also been identified and implicated in the pathology of PD. ${ }^{[32-38]}$ At the same time, $\alpha$-syn and its PTMs have been detected as potential biomarkers of PD in blood erythrocytes ${ }^{[39]}$ and blood plasma. ${ }^{[40,41]}$ The detection of these PTMs have so far been based mainly on immunoblotting. This approach can detect only single PTM occurring with relatively high abundance, which is an acknowledged limitation of immunoblotting. ${ }^{[42]}$ The latter authors also discussed the important role of mass spectrometry (MS) in $\alpha$-syn PTM detection, highlighting attributes of MS include its high resolution, performance, and productivity. Our experience indicates that MS can detect multiple PTMs including low abundant PTM forms from one single injection. However, for very low abundance, as

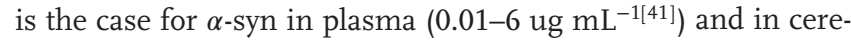
brospinal fluid (CSF; approximately $50 \mathrm{pg} \mathrm{mL}^{-1[43]}$ ), enrichment is necessary for MS identification and quantification.

Here, we examined the $\alpha$-syn dopaminylation from blood plasma and its correlation with PD. We first simulated the reaction of DA and $\alpha$-syn in vitro, and observed that DA could induce $\alpha$-syn oligomerization. We also analyzed the biochemical mechanism and modification forms using high resolution MS. Furthermore, we aimed to detect endogenous forms of modified $\alpha$-syn. This was accomplished using immunoprecipitation (IP) coupled with MS using blood plasma from PD patients and healthy control (HC) subjects. IP enriched the low level of endogenous plasma $\alpha$-syn. High resolution Orbitrap Elite MS (Thermo Fisher Scientific, Waltham, MA, USA) was applied to detect DA modification of $\alpha$-syn. At the same time, other conventional PTM forms of $\alpha$-syn, including those present in low levels, were also analyzed.

\section{Results}

\subsection{DA Induces $\alpha$-syn Oligomerisation In Vitro by Adding its Oxidative Derivatives into Lysine Sites of $\alpha$-syn}

DA modification forms, predicted to arise from $\alpha$-syn treatment with DA and the DA derivatives, are summarized from articles

\section{Clinical Relevance}

DA toxicity on $\alpha$-syn has been linked to the degeneration of dopaminergic neurons in PD, the toxic mechanism has so far been investigated only in in-vitro studies. The toxic mechanism study with clinical samples is needed to validate for understanding PD pathology. This study reports an optimized protocol for $\alpha$-syn extraction and detection in clinical plasma samples, which allows the identification of multiple PTMs, especially dopaminylation of $\alpha$-syn that are at present difficult to be identified in conventional immunoblotting due to the lack of suitable antibodies and the relatively low abundance of plasma $\alpha$-syn compared to central nervous system (CNS). To our knowledge, it is the first detection of endogenous $\alpha$-syn dopaminylation (identification and quantification) in both $\mathrm{HC}$ and PD blood plasma. Since blood samples are much more readily accessible and less invasive than cerebrospinal fluid (CSF) samples, this opens up a novel avenue for clinical detection and monitoring of dopaminylation of $\alpha$-syn, which is a key protein marker for PD study.

as shown in Figure 1 and Table 1. The results from the simulated reaction and detected modification mechanism is integrated in the third form. DA could be oxidized into DAQ with a 2 Da mass loss in vitro or in vivo, followed by the transformation of DAQ to DC with an additional 2 Da mass loss (Figure 1A). The in vitro derivatives of DA were detected with LC-MS; DAQ and DC were the main products of DA oxidation (Figure S2, Supporting Information). These DA derivatives were similar in the presence of quinone groups (Figure 1B), which is the active group reacting with the side chain amino group of lysine in $\alpha$-syn (Figure $1 \mathrm{C}$ ). This reaction releases $\mathrm{H}_{2} \mathrm{O}$, which is different from the traditional monoaminylation on glutamine with the release of $\mathrm{NH}_{3}{ }^{[44,45]}$ and the addition of DAQ on cysteine. ${ }^{[46]}$ With the catalytic action of monoamine oxidase (MAO), DA can be oxidized to DOPAL, which is the most toxic derivative of DA (Figure 1A).

The reaction between DA and $\alpha$-syn was simulated in vitro using DA and recombinant $\alpha$-syn in a $37{ }^{\circ} \mathrm{C}$ water bath, as shown in Figure 2. DA-induced $\alpha$-syn oligomerization (Figure 2A) in a concentration-dependent manner (Figure S3A, Supporting Information). This effect was inhibited by antioxidants, such as ascorbic acid and dithiothreitol (DTT) (Figure 2B). These findings suggested that DA-induced $\alpha$-syn oligomerization depending on the oxidation of DA, which was confirmed as the oxidation of methionine and addition of DA oxidative derivative DAQ and DC in $\alpha$-syn (Figure 2C,D). The MS/MS results revealed the addition of DA derivatives in the lysine sites of $\alpha$-syn (Figures S3B and S3C, Supporting Information). MS intensities were used to quantify the modification ratio, which demonstrated the prevalence of DA-mediated methionine oxidation (modification ratio of 0.741 ). The addition of DA derivatives resulted in a relatively low ratio $(0.019-0.110)$. The findings indicated that the in vitro biochemical mechanism involved DA induced $\alpha$-syn oligomerization that occurred due to altered structure of $\alpha$-syn, including oxidation of amino acids of $\alpha$-syn and the addition of DA derivatives to the lysine sites of $\alpha$-syn, for which DA oxidation was necessary. 


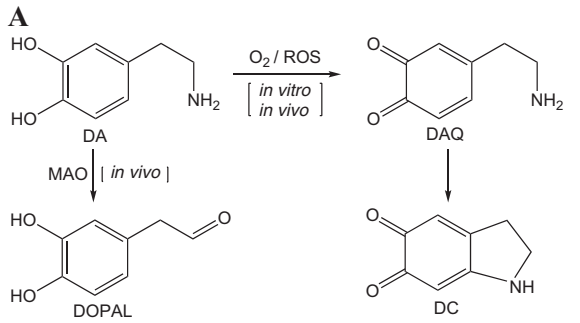

$\mathrm{R}=\mathrm{O}=2$
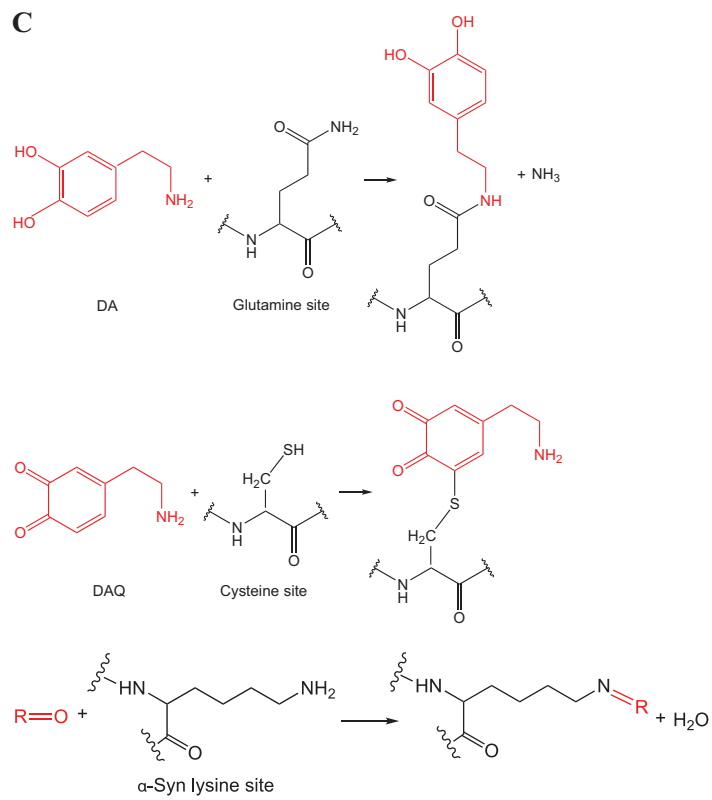

Figure 1. Predicted modification mechanism shown in reaction formula. A) The oxidation of DA and generation of DA derivatives in vitro and in vivo. The reaction conditions are above and below the arrows. B) The active quinone group of three DA derivatives. C) Predicted reaction mechanism for that DA derivatives modify $\alpha$-syn, within which the third form is validated by current study.

\subsection{Endogenous $\alpha$-syn Dopaminylation Is Present in PD and HC, and the Level of One Dopaminylation Peptide from $\alpha$-syn is Significantly Higher in PD Patients Compared with HC Subjects}

To detect $\alpha$-syn dopaminylation in blood plasma of PD patients and to assess whether dopaminylation plays a role for $\alpha$-syn based neurodegeneration, it was necessary to successfully detect low levels of $\alpha$-syn in blood plasma. For this, we used magnetic beads bound to antibody for full-length human $\alpha$-syn to capture and enrich plasma $\alpha$-syn. The detected plasma $\alpha$-syn peptides are summarized in Table 2. Similar to the in vitro derivatives, we predicted that the toxicity of DOPAL would also be based on its quinone group that reacts with the amino group of lysine. This was subsequently proven by endogenous $\alpha$-syn PTM identification with blood plasma samples.

The detection of endogenous $\alpha$-syn in PD patients involved the examination of blood plasma from age and gender matched people without neurodegenerative conditions ( $\mathrm{HC})$, and individuals with major depressive disorder (MDD) as disease control. Analysis involved nano LC-MS followed by MaxQuant and QI. With the txt files exported from QI, abundances of $\alpha$-syn and $\alpha$-syn modifications for the three groups were plotted (Figure 3 ). The $\alpha$-syn level in plasma of PD patients was comparable with the level in $\mathrm{HC}$ subjects, but was significant higher in the plasma of MDD patients compared with HC subjects (Figure 3A). The average level (mean level of all DA modified peptide in each pool) of $\alpha$-syn dopaminylation in the three groups was not significantly different (DAQ modification in Figure 3B and DOPAL modification in Figure $3 \mathrm{C}$ ). However, quantification of DA modified $\alpha$-syn individual peptides revealed that the level of peptide with the ${ }^{34} \mathrm{~K}$ DAQ addition was significantly higher in PD and MDD patients (Figure 3D). To better understand $\alpha$-syn dopaminylation and other conventional PTMs, a modification map was constructed based on the identification results (Figure 3F). All possible modification sites are denoted by a pair of red brackets with the modification probability calculated by MaxQuant. The map revealed that active sites for modification resided mainly in the amphipathic lysine-rich $\mathrm{N}$-terminal of $\alpha$-syn. Dopaminylation was distributed among ${ }^{6} \mathrm{~K},{ }^{10} \mathrm{~K},{ }^{12} \mathrm{~K},{ }^{23} \mathrm{~K},{ }^{32} \mathrm{~K},{ }^{34} \mathrm{~K},{ }^{43} \mathrm{~K}$, and ${ }^{45} \mathrm{~K}$, among which ${ }^{23} \mathrm{~K},{ }^{43} \mathrm{~K}$, and ${ }^{45} \mathrm{~K}$ displayed high probability $(0.990-1.000)$ with the remaining probabilities from $0.464-0.486$. Ubiquitination was the most common modification, consistent with previous blood $\alpha$-syn PTM analysis. ${ }^{[40]}$ Most ubiquitination probabilities exceeded 0.757 (0.464 for ${ }^{10} \mathrm{~K}$ and $0.757-1.000$ for the rest). Nitration and phosphorylation were found in only one site each with high probabilities (1.000 for ${ }^{39} \mathrm{Y}$ nitration and 0.996 for ${ }^{22} \mathrm{~T}$ phosphorylation).

Table 1. Summary of the main forms of DA modification. DA (dopamine); DAQ (dopamine-quinone); DC (dopaminochrome); DOPAL (3,4Dihydroxyphenylacetaldehyde).

\begin{tabular}{|c|c|c|c|c|c|}
\hline Item & $\begin{array}{l}\text { Added } \\
\text { molecular }\end{array}$ & $\begin{array}{l}\text { Release } \\
\text { elements }\end{array}$ & $\begin{array}{l}\text { Added } \\
\text { Mass }\end{array}$ & $\begin{array}{l}\text { Modified } \\
\text { residue }\end{array}$ & Reference basis \\
\hline (1) & $+\mathrm{DA}$ & $-\mathrm{NH}_{3}$ & 136.0524 & Glutamine (Q) & ${ }^{[44]}$ L. A. Farrelly, 2019, Nature; ${ }^{[45]}$ I. S. Maze, 2016, Alcoholism (NY) \\
\hline (2) & + DAQ & l & 151.0633 & Cysteine (C) & ${ }^{[46]}$ R. E. Whitehead, 2001, Journal of Neurochemistry \\
\hline \multirow[t]{3}{*}{ (3) } & $+\mathrm{DAQ}$ & $-\mathrm{H}_{2} \mathrm{O}$ & 133.0528 & Lysine (K) & [20] C. Follmer, 2015, Journal of Biological Chemistry \\
\hline & + DOPAL & $-\mathrm{H}_{2} \mathrm{O}$ & 134.0368 & & \\
\hline & $+\mathrm{DC}$ & $-\mathrm{H}_{2} \mathrm{O}$ & 131.0371 & & \\
\hline
\end{tabular}


A

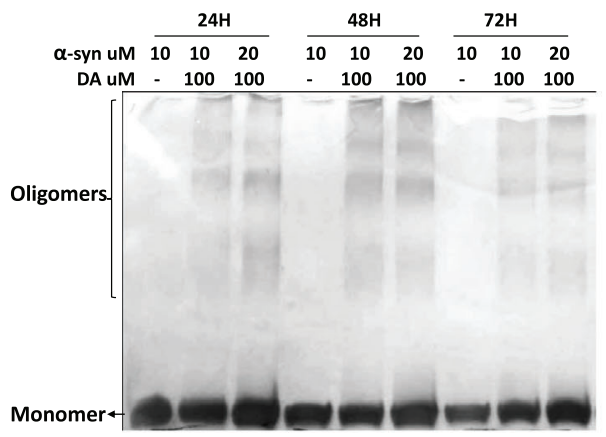

B

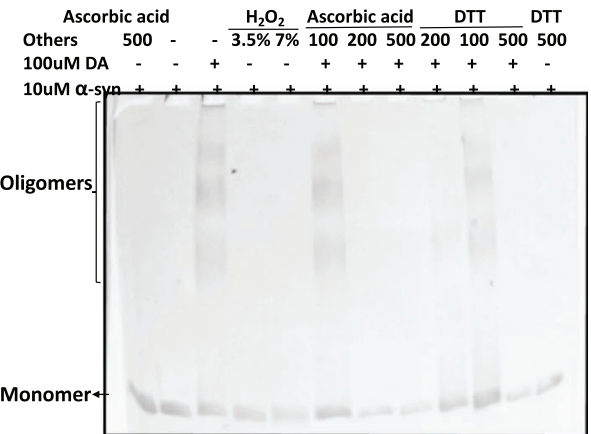

C

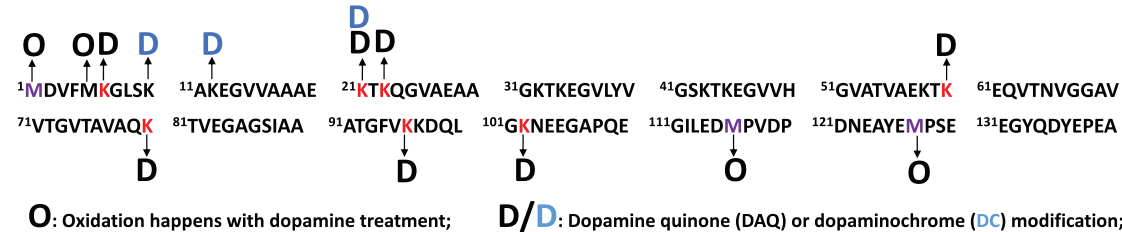

D

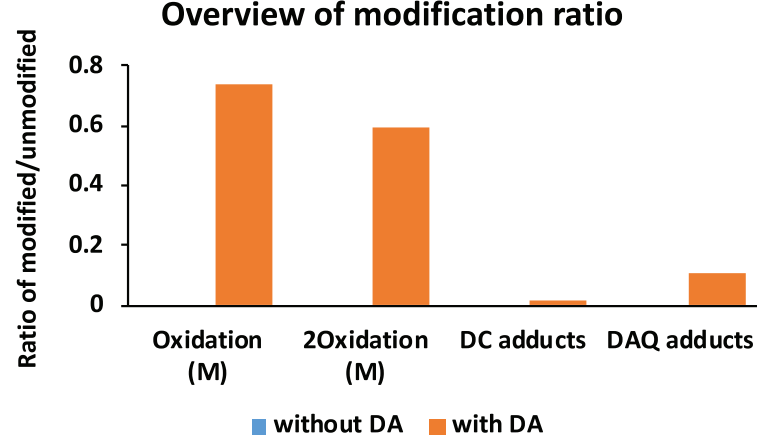

Figure 2. The forms of $\alpha$-syn modified by DA revealed by in vitro test. A) DA induces $\alpha$-syn forming stable oligomers. 10 and $20 \mu \mathrm{M} \alpha$-syn are treated without or with $100 \mu \mathrm{m} \mathrm{DA}$ and incubate at $37^{\circ} \mathrm{C}$ for $24 / 48 / 72 \mathrm{~h}$. B) DA induced $\alpha$-syn oligomerization depends on DA's oxidation. Fixed concentration of $\alpha$-syn $(10 \mu \mathrm{M})$ and DA $\left(100 \mu \mathrm{M}\right.$ final concentration) are used. The final concentrations of $\mathrm{H}_{2} \mathrm{O}_{2}$ are shown on top of the lanes. All of the samples were incubated for $48 \mathrm{~h}$ at $37^{\circ} \mathrm{C}$. C) The modification map. With the treatment of DA, there are the oxidation of methionine (M) detected. At the same time, the adducts of $\alpha$-syn with dopamine quinone (DAQ) or dopaminochrome (DC) were also detected. From the modification map, one can see each modification and its site. Specially, DAQ addition happens on ${ }^{6} \mathrm{~K},{ }^{21} \mathrm{~K},{ }^{22} \mathrm{~K},{ }^{60} \mathrm{~K},{ }^{70} \mathrm{~K},{ }^{96} \mathrm{~K},{ }^{102} \mathrm{~K}$, and DC modification happens on ${ }^{10} \mathrm{~K},{ }^{12} \mathrm{~K},{ }^{21} \mathrm{~K}$. D) The ratios of all modifications. The $x$-axis shows different kind of modifications that DA induced on $\alpha$-syn; the $\gamma$-axis shows the modifying level by using the average ratio that the intensity of modified peptide divided by the intensity of corresponding unmodified peptide.

The IP-MS based strategy detected all possible dopaminylation sites and other conventional PTMs in one single experiment in a high-throughput manner. Besides dopaminylation, we could also identify and quantify other conventional PTMs, such as methionine oxidation, acetylation, phosphorylation, and ubiquitination. For the ${ }^{23} \mathrm{~K}$ ubiquitination was significantly higher in PD and MDD patients compared with HC subjects (Figure 3E). Interestingly, the dopaminylation level of $\alpha$-syn in MDD patients was even higher than in PD patients, indicating higher level of DA toxicity in MDD. Thus, DA toxicity $\alpha$-syn was evident in vitro and in endogenous plasma. This indicates a role of $\alpha$-syn dopaminylation in $\alpha$-syn structural modification and aggregation.

\subsection{Dopaminylation Levels of $\alpha$-syn Interacting Proteins Different in HC, PD, and MDD Subjects}

Besides PTMs, $\alpha$-syn interaction with proteins influence formation of $\alpha$-syn, $\alpha$-syn aggregation, and other pathologies of PD. ${ }^{[47,48]}$ The Uniprot database contains fourteen $\alpha$-syn interacting proteins, which could be downloaded together with $\alpha$ syn when we searched the database for $\alpha$-syn. Since the interacting protein complex might be immunoprecipitated using the magnetic beads, the LC-MS raw data was identified by matching the downloaded $\alpha$-syn database with the interacting proteins. Some proteins could be identified from the exported MaxQuant file. Three peptides displayed different levels of dopaminylation 
Table 2. Information of unmodified/modified $\alpha$-syn peptides from IP elution in blood plasma.

\begin{tabular}{|c|c|c|c|c|c|}
\hline Modification & Sequence & $\mathrm{m} / \mathrm{z}$ & $\begin{array}{l}\text { Retention } \\
\text { time [min] }\end{array}$ & Accurate Mass & Charge \\
\hline Unmodified & _AKEGVVAAAEK_ & 536.8031 & 23.5 & 1071.5917 & 2 \\
\hline Unmodified & _AKEGVVAAAEKTK_ & 651.3768 & 31.3 & 1300.7391 & 2 \\
\hline Unmodified & _EGVVAAAEK_ & 437.2363 & 37.5 & 872.4580 & 2 \\
\hline Unmodified & _EGVVAAAEKTK_ & 551.7977 & 26.8 & 1101.5808 & 2 \\
\hline Unmodified & _EQVTNVGGAVVTGVTAVAQK_ & 643.3535 & 29.9 & 1927.0388 & 3 \\
\hline Unmodified & _EQVTNVGGAVVTGVTAVAQK_ & 964.5314 & 35.8 & 1927.0482 & 2 \\
\hline Unmodified & _GLSKAKEGVVAAAEK_ & 486.6153 & 29.7 & 1456.8241 & 3 \\
\hline Unmodified & _QGVAEAAGK_ & 415.7232 & 23.5 & 829.4319 & 2 \\
\hline Unmodified & _TKEQVTNVGGAVVTGVTAVAQK_ & 719.7332 & 32.4 & 2156.1779 & 3 \\
\hline Unmodified & _TKEQVTNVGGAVVTGVTAVAQK_ & 1079.0936 & 37.4 & 2156.1726 & 2 \\
\hline Unmodified & _TVEGAGSIAAATGFVK_ & 739.8965 & 33.8 & 1477.7785 & 2 \\
\hline Unmodified & _TVEGAGSIAAATGFVK_ & 493.5860 & 30.8 & 1477.7361 & 3 \\
\hline Unmodified & _TVEGAGSIAAATGFVKK_ & 536.2809 & 27.2 & 1605.8210 & 3 \\
\hline Unmodified & _TVEGACSIAAATGFVKK_ & 803.9426 & 31.2 & 1605.8707 & 2 \\
\hline Unmodified & _EGVLYVGSK_ & 476.2616 & 28.9 & 950.5086 & 2 \\
\hline Unmodified & _EGVLYVGSKTK_ & 590.8324 & 33.4 & 1179.6502 & 2 \\
\hline Unmodified & _EGVVHGVATVAEK_ & 432.5700 & 28.7 & 1294.6883 & 3 \\
\hline Unmodified & _EGVVHGVATVAEK_ & 648.3512 & 26.2 & 1294.6879 & 2 \\
\hline Unmodified & _EGVVHGVATVAEKTK_ & 508.9476 & 28.2 & 1523.8209 & 3 \\
\hline Unmodified & $\begin{array}{l}\text { _EGVVHGVATVAEKTKEQVTNVGG } \\
\text { AVVTGVTAVAQK_ }\end{array}$ & 1145.2850 & 42.2 & 3432.8332 & 3 \\
\hline Unmodified & _TKEGVLYVGSK_ & 590.8324 & 33.4 & 1179.6502 & 2 \\
\hline Unmodified & _TKEGVLYVGSK_ & 394.2243 & 33.4 & 1179.6512 & 3 \\
\hline Unmodified & _TKEGVVHGVATVAEK_ & 508.9476 & 28.2 & 1523.8209 & 3 \\
\hline Unmodified & _TKEGVVHGVATVAEK_ & 762.9173 & 28.2 & 1523.8201 & 2 \\
\hline Unmodified & _TKEGVVHGVATVAEKTK_ & 585.3306 & 30.6 & 1752.9700 & 3 \\
\hline Unmodified & _KDQLGK_ & 688.3983 & 27.1 & 687.3910 & 1 \\
\hline Ubiquitination & _TK(ubiquitination)QGVAEAAGK & 391.8806 & 27.5 & 1172.6199 & 3 \\
\hline $\begin{array}{l}\text { DAQ- } \mathrm{H}_{2} \mathrm{O} \text {, phosphorylation, } \\
\text { ubiquitination, }\end{array}$ & $\begin{array}{c}\mathrm{T} \text { (phosphorylation) } \mathrm{K}\left(\mathrm{DAQ}-\mathrm{H}_{2} \mathrm{O}\right) \\
\text { EGVLYVVAEK (ubiquitination) }\end{array}$ & 831.9577 & 35.7 & 1661.9009 & 2 \\
\hline
\end{tabular}

between PD and HC subjects (Figure 4). An FAK2 (Proteintyrosine kinase 2-beta) peptide had a lower level of dopaminylation in PD patients compared with HC subjects. VPS41 (Vacuolar protein sorting-associated protein 41 homolog) and SKAP2 (Src kinase-associated phosphoprotein 2) peptides had a higher level of dopaminylation in PD patients compared with HC subjects. These differences suggested the relevance of dopaminylation in $\alpha$-syn aggregation or other PD pathologies. Compared with HC, the changes of these three proteins in MDD are not significant: $\mathrm{p}$ value 0.13 for VPS41, 0.14 for SKAP2, 0.98 for FAK2. The insignificance meant that the dopaminylation on $\alpha$-syn interacting proteins are specific for PD compared with both healthy control (HC group) and disease control (MDD group).

\section{Discussion}

While PD and MDD are two quite different types of diseases, they both are found linked to dopaminergic hypofunction. ${ }^{[49,50]}$ Oxidative stress might be one of the cause responsible for this hypofunction. ${ }^{[51,52]}$ If so, it at least partially explain why we find abnormal $\alpha$-syn dopaminylation in the plasma of PD and MDD patients. This is very interesting, but need future cellular or animal level verification. It worth note that 16 patients took levodopa among the collected 30 PD patients. They are the majority of patients in the clinic. As our in vitro result showed that the $\alpha$-syn dopaminylation was happening based on dopamine oxidation rather than dopamine itself. If an increase in $\alpha$-syn dopaminylation is observed, it more implies the increase in oxidation stress issue rather than the increase in the level of dopamine itself, although they have links. We think the abnormal dopaminylation of $\alpha$-syn is highly possible caused by the stress body condition of PD patients. In addition, the level of $\alpha$-syn dopaminylation between PD (16 of 30 with uptake of levodopa) and MDD (28 without uptake of levodopa) are in the similar level, reflecting that a disease indeed could be a cause responsible for the increase in $\alpha$ syn dopaminylation. Anyway, we could not exclude the role of levodopa. An examination using a larger amount of participants in the future, especially newly diagnosed participants, would solve this concern. 
A

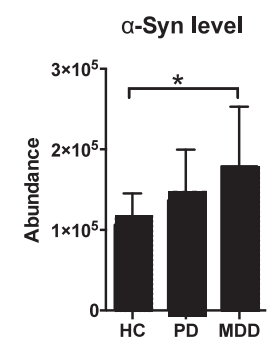

D

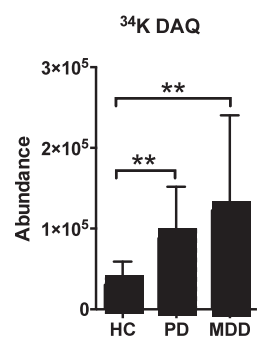

B

a-Syn DAQ average

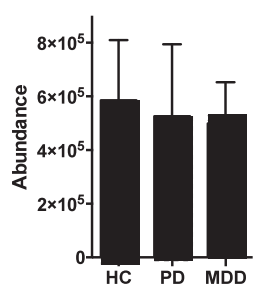

C

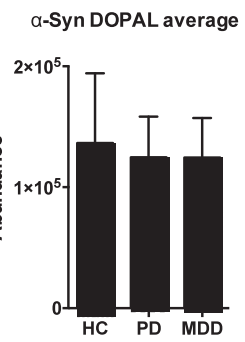

$\mathbf{E}$

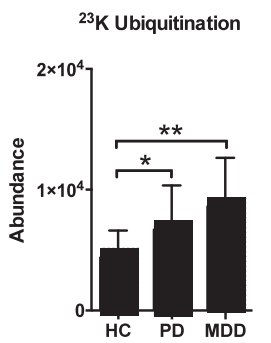

F

${ }^{1}$ Ace-M (Oxi_1) DVFM (Oxi_1) K(DOPAL_1;Ubi_0.757)GLSK(DOAPL_0.479;Ubi_1) ${ }^{11}$ AK(DOAPL_0.479;Ubi_1)EGVVAAAE ${ }^{21} \mathrm{~K}$ (Ubi_0.964) T (Pho_0.996) K(DOAPL_0.99)QGVAEAA ${ }^{31}$ GK(DAQ_0.483; DOPAL_0.486; Ubi_0.979)TK (DAQ_0.482; DOPAL_0.486) EGVLY (Nit_1) V ${ }^{41}$ GSK (DAQ_1; Ubi_0.965) TK (DAQ_1) EGVVH ${ }^{51}$ GVATVAEKTK(Ubi_0.794) ${ }^{61}$ EQVTNVGGAV ${ }^{71}$ VTGVTAVAQK ${ }^{81}$ TVEGAGSIAA ${ }^{91}$ ATGFVKKDQL ${ }^{101}$ GKNEEGAPQE ${ }^{111}$ GILEDMPVDP ${ }^{121}$ DNEAYEMPSE ${ }^{131}$ EGYQDYEPEA

Figure 3. Quantification of blood plasma $\alpha$-syn and its modification. The mean value of abundance in each group with their SD values is shown in the histogram. The $t$-test $p$ value is showing above the comparison line among every two groups, $*$ refers to $t$-test $p$ value $<0.05$, $* *$ refers to $t$-test $p$ value $<0.01$. A) The comparison of $\alpha$-syn level among three groups. The abundance of $\alpha$-syn in each pool is determined by the average intensity of its top three abundant unmodified peptides. B,C) The comparison of $\alpha$-syn modification level among three groups. B) the average intensity of DAQ modified peptide fragments are compared; C) the average intensity of DOPAL modified peptide fragments are compared. D,E) The quantification plots of two significant changed peptides, which are corresponding to the peptides in Table 2. The intensities used are the exported intensity of each peptide from each pool from QI. D) DAQ modified $\alpha$-syn peptide, sequence_T(Phosphorylation)K(DAQ- $\mathrm{H}_{2}$ O) EGVLYVVAEK(Ubiquitination)_. E) Ubiquitination modified $\alpha$-syn peptide, sequence _TK(Ubiquitination)QGVAEAACK_. F) The modification map for detected PTMs of $\alpha$-syn from human blood plasma. The probability of each PTM is shown in a pair of brackets followed by its modification site. For example, ${ }^{6} \mathrm{~K}$ (DOPAL_0.464) means the DOPAL-H ${ }_{2} \mathrm{O}$ modification in the ${ }^{6} \mathrm{~K}$ site of $\alpha$-syn has the probability of 0.464 according to the exported result of evidence file from MaxQuant.

A

FAK2

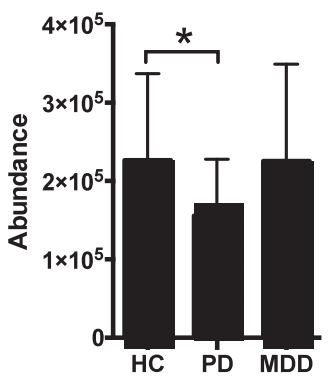

B

VPS41

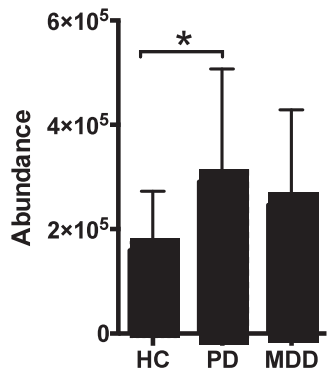

C

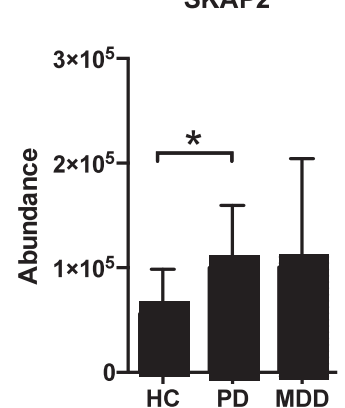

Figure 4. Quantification for modified peptides of $\alpha$-syn interacting proteins from digested IP elution in blood plasma that are with significant difference between HC and PD. The quantification plots of three significant changed peptides from $\alpha$-syn interacting proteins. The intensities used are the exported intensity of each peptide from each pool from QI. The mean value of abundance in each group with their SD values is shown in the histogram. The $t$-test $p$ value is showing above the comparison line among every two groups, $*$ refers to $t$-test $p$ value $<0.05$. A) DAQ and DOPAL modified FAK2 peptide, sequence_(Acetyl)NAR(DAQ_0.500)YR(DOPAL_0.500)TPK_. B) DOPAL modified VPS41 peptide, sequence:_(Acetyl)DHLK(DOPAL_0.656)K(Ubiquitination_0.656)DSQNK_. C) DAQ and DOPAL modified SKAP2 peptide, sequence _EK(Ubiquitination_0.998)R(DOPAL_0.998)ES(Phosphorylation_1.000)LIK(DAQ_0.404)KIK(DOPAL_0.618)_. 
PTMs have emerged as important determinants of $\alpha$ syn physiological and pathological functions, and potential biomarkers of PD and other synucleinopathies. ${ }^{[42]}$ Among the PTMs, dopaminylation of $\alpha$-syn is of particular interest as it is related to the dopaminergic selectivity of $\alpha$-syn contributing to neurotoxicity observed in PD. ${ }^{[53]}$ Detection of $\alpha$-syn PTMs in blood plasma remains challenging because of the low abundance of $\alpha$-syn and $\alpha$-syn PTMs and the necessity of careful antibody selection. Our IP-MS strategy detected both dopaminylation and conventional PTMs in a single experiment, applying selectivity extraction of $\alpha$-syn and the excellent detection sensitivity and high-throughput capability of MS analysis.

In our study, the plasma $\alpha$-syn level in PD patients was not significantly increased compared to HC subjects ( $t$-test $p$ value 0.12 ), consistent with the total $\alpha$-syn level finding from a previous study with plasma. ${ }^{[1]}$ Another longitudinal study concluded that the total $\alpha$-syn level in plasma increased with time for up to 20 years after the initial appearance of PD symptoms. ${ }^{[40]}$ Thus the reason why detected $\alpha$-syn level in plasma was not significantly increased in PD patients compared with $\mathrm{HC}$ subjects in present study might be that PD patients were with average duration of 3.9 years (short disease duration). Examining the CNS, CSF level of $\alpha$-syn has been shown to increase in the later phase, corresponding to five years after symptom onset, but remain stable in the earlier symptomatic phase of PD. ${ }^{[54]}$ However, obtaining blood is less traumatic than CSF for patients. Further studies involving more subjects will help clarify the value of this strategy.

The present study shows two key factors of DA induced $\alpha$ syn oligomerisation (DA toxicity) from in vitro analysis, $\alpha$-syn dopaminylation and DA induced $\alpha$-syn methionine oxidation. Dopaminylation is a direct evidence of the structure change of $\alpha$-syn in PD. Oxidation of four methionine residues, distributed in the N-terminal and C-terminal regions of $\alpha$-syn, can change the polarity of $\alpha$-syn, influence their end-to-end association ${ }^{[55]}$ and inhibit the propensity of $\alpha$-syn to adopt an ordered secondary structure, ${ }^{[56]}$ which increases the formation of $\alpha$-syn oligomers. The present data reveal different forms of DA-induced modification of $\alpha$-syn in vitro and in vivo. Firstly, endogenous dopaminylation sites on $\alpha$-syn are less frequent than in vitro reacted samples, which may due to the higher concentration of DA and much simpler reacting condition in vitro. Secondly, methionine oxidation displayed the highest modification ratio among all types of modifications in vitro, consistent with other studies. ${ }^{[23,55,57]}$ However, methionine oxidation was not stable detected in blood plasma in present study. Thus, results obtained from in vitro experiments do not fully or accurately predict the situation on a whole organism, indicating the importance of analysis based on clinical samples.

In the CNS, the endogenous DA and other monoamines serve as neurotransmitters as well as bind with neuronal and glial proteins, such as histone ${ }^{[4]}$ and fibronectin, ${ }^{[58]}$ catalyzed by transglutaminase. ${ }^{[59]}$ Different from dopaminylation here that the quinone group of the DA derivatives (DAQ, DC, and DOPAL) react with the side chain amino-group of lysine with the release of $\mathrm{H}_{2} \mathrm{O}$ molecule, monoaminylation has been defined before that the amino-group of monoamines react with the side chain amino-group of glutamine in the protein sequence with the release of $\mathrm{NH}_{3}$ molecule. Monoaminylation has been proposed as a novel form of protein $\mathrm{PTM},{ }^{[60]}$ including histaminylation, ${ }^{[61,62]}$ serotonylation, ${ }^{[44]}$ dopaminylation, ${ }^{[45]}$ and norepinephrinylation. ${ }^{[63]}$ Besides our finding that dopaminylation may play a role in PD pathology, other monoaminylation forms are reported to be related with important biological processes ${ }^{[64,65]}$ and disease development. ${ }^{[6]}$ So protein monominylation should be paid continuous attention.

VSP41 is protective against both $\alpha$-syn and neurotoxicmediated injury invertebrate and cellular models of PD. These protective functions may be related to enhanced clearance of misfolded or aggregated protein, including $\alpha$-Syn. VSP41 is identified as a potential therapeutic target for PD. ${ }^{[67]}$ SKAP2, and FAK2 are both involved in the phosphorylation of $\alpha$-syn. SKAP2 is in the Src family kinases in the CNS, which is involved in $\alpha$-syn phosphorylation. ${ }^{[68]}$ FAK2/RAFTK (related adhesion focal tyrosine kinase)-associated protein regulates $\alpha$-syn phosphorylation following cell stress. Hyperosmotic stress induced phosphorylation of tyrosine 125 of $\alpha$-syn via FAK2/RAFTK, which acted through Src family kinases. ${ }^{[69]}$ The significant different level of these three proteins' dopaminylation in PD compare with HC shows that dopaminylation affects the clearance of abnormal $\alpha$ syn and the phosphorylation process of $\alpha$-syn in PD.

The collective findings indicate the value of the combination of IP and MS for detection of plasma $\alpha$-syn and related PTMs, especially dopaminylation. The strategy may help clarify the role of dopaminylation in PD. Endogenous dopaminylation detection from biofluids and tissues could be feasible with the present method, since there is no dopaminylation specific antibody that could be applied for immunoblotting. In addition, other PTM forms could be detected than is possible using immunoblotting, which will be valuable in discovering more pathological evidence for PD and other neurodegenerative diseases.

\section{Experimental Section}

DA and $\alpha$-syn Reaction In Vitro: DA hydrochloride, L-ascorbic acid, DL-dithiothreitol (DTT), and iodoacetamide (IAA) were purchased from Sigma-Aldrich (St. Louis, MO, USA). Purified ( $\geq 90 \%)$ recombinant human $\alpha$-syn with an $\mathrm{N}$-terminal histidine tag (Sigma-Aldrich) was used for expression in Escherichia coli. Protein samples were prepared by diluting $\alpha$-syn to a final concentration of $10 \mu \mathrm{M}$ and adding dilute DA or antioxidants to final concentrations in distilled water and incubating at $37^{\circ} \mathrm{C}$ for ideal time (concentration and incubations could be seen from the figures). Each sample obtained during preparation was analyzed using both liquid chromatography (LC)-MS and sodium dodecyl sulfate-polyacrylamide gel electrophoresis (SDS-PAGE). For SDS-PAGE, five microliters of each incubated sample was mixed with an equal volume of loading buffer and the total volume of $10 \mu \mathrm{L}$ was loaded onto a $10 \%$ mini-gel $(0.75 \mathrm{~mm}$ thick). After loading, the two gels that were run in a tank that received a running voltage of $120 \mathrm{~V}$. Optimised silver staining and photography were done following electrophoresis.

Plasma $\alpha$-syn Extraction Using IP: Clinical plasma samples (30 PD, 30 $\mathrm{HC}, 28$ major depressive disorder [MDD]) were collected according to the standard operating procedure with the ethical proof from Dalian Central Hospital, Dalian, China. Blood was collected at 6:00 AM. Patients had not consumed food or water for the night preceding blood collection at Dalian Central Hospital (for PD and HC) and Dalian Seventh People's Hospital (for MDD). The blood was immediately centrifuged to obtain plasma, which was portioned and stored at $-80{ }^{\circ} \mathrm{C}$ within an hour. PD patients were diagnosed according to MDS-Unified Parkinson's Disease Rating Scale (MDS-UPDRS) criteria. There are 16 male subjects and 14 female subjects in PD group with the average age 69.8 (49-81). According 
to Hoehn-Yahr $(\mathrm{H}-\mathrm{Y})$ staging in $1967,{ }^{[70]}$ there are $53 \%(16 / 30)$ in stage $\mathrm{H}-\mathrm{Y} 2,37 \%(11 / 30)$ in stage $\mathrm{H}-\mathrm{Y} 1,7 \%(2 / 30)$ in stage $\mathrm{H}-\mathrm{Y} 4,3 \%(1 / 30)$ in stage $\mathrm{H}-\mathrm{Y} 3$. The $\mathrm{HC}$ subjects were healthy individuals or patients who suffered from headache, trigeminal neuralgia, dizzy, vertigo, or occipital neuralgia. There are 13 male subjects and 17 female subjects in $\mathrm{HC}$ group with the average age $41.0(22-80)$. MDD patients were diagnosed according to the Hamilton depression scale (HAMD) with value ranged from 18 to 37 . There are 12 male subjects and 16 female subjects in MDD group with the average age 59.0 (35-78). For the stage of MDD patients, six are mild, 14 are moderate, six are severe, and two are unknown.

The plasma pool consisted with two $500 \mu \mathrm{L}$ blood plasma samples from two participants in one group. The priority of pooling was between one male participant and one female participant. This approach was adopted to decrease within-group variances caused by gender difference and to reach a desired plasma proteome coverage within the available measuring time. ${ }^{[71]}$ Very large pools were rejected since they introduced unacceptable variances for the statistical analysis. ${ }^{[72]}$ Nine hundred microliters of each pool was treated with $80 \mu \mathrm{L}$ resin from an anti-human $\alpha$-syn immunomagnetic beads kit (Sino Biological, Beijing China; Cat. No. MB12093-R017-1). The IP procedure was conducted according to the manufacture's protocol. Briefly, $80 \mu \mathrm{L}$ of a suspension of beads was washed twice by 150 and $1000 \mu \mathrm{L}$ of $1 \times$ Tris-buffer with Tween (TBST) in a $1.5 \mathrm{~mL}$ microcentrifuge tube. Five hundred microliters of plasma and $80 \mu \mathrm{L}$ of $5 \times$ TBST was added to the beads, followed by gentle stirring for $30 \mathrm{~min}$. The beads were collected using a magnetic separator, washed twice with $300 \mu \mathrm{L}$ of $5 \times$ TBST twice then gently washed with $300 \mu \mathrm{L}$ of water. Finally, the beads were eluted with $50 \mu \mathrm{L}$ acidic elution buffer for $5 \mathrm{~min}$ at room temperature for trypsin digestion.

Trypsin Digestion and Stage Tip Desalting: Trypsin (1 ug, Roche, Switzerland) was added to each reacted in vitro sample or plasma collected IP sample and incubated at $37^{\circ} \mathrm{C}$ overnight (16-18 h). Digested samples were then purified using stage tips ${ }^{[73]}$ before liquid chromatography-mass spectrometry (LC-MS) analysis. Briefly, every step was conducted by centrifuging at $2000 \mathrm{~g}$ and $4{ }^{\circ} \mathrm{C}$. The stage tips were ac tivated with $200 \mu \mathrm{L} \mathrm{LC}$ solvent B $(80 \%$ acetonitrile containing $0.2 \%$ formic acid) twice and balanced with $200 \mu \mathrm{L} L C$ solvent $\mathrm{A}$ ( $2 \%$ acetonitrile containing $0.2 \%$ formic acid) twice. The digested sample was loaded into the stage tip and washed with $100 \mu \mathrm{L}$ LC solvent A then eluted with $200 \mu \mathrm{L}$ LC solvent $B$. The elution fraction was collected into one fresh tube, lyophilized and dissolved in $30 \mu \mathrm{L} \mathrm{LC}$ solvent A for LC-MS analysis.

Nano LC-MS Data Acquisition: All reacted in vitro samples were analyzed using a Thermo Dionexnano LC system coupled with a Thermo XL MS and Discovery Orbitrap-MS (all from Thermo Fisher Scientific). For the nano LC-MS analysis, an in-house-packed C18 (ReproSil-Pur, C18, $3 \mu \mathrm{m}$ ) nano column $(75 \mu \mathrm{m}$ id $\times 20 \mathrm{~cm}, 10 \mu \mathrm{m}$ tip; New Objective, Woburn, MA USA) was used. The column temperature was maintained at $25^{\circ} \mathrm{C}$, and the injection volume was $5 \mu \mathrm{L}$. The separation was performed using a $100 \mathrm{~min}$ gradient program with a mix of solvent $A$ and solvent $B$. The pump flow

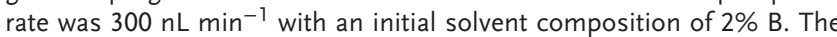
gradient was conducted from $2 \%$ to $10 \%$ B over 15 min (balancing time), from $10 \%$ to $30 \% \mathrm{~B}$ for 40 min (low eluting time), from $30 \%$ to $60 \% \mathrm{~B}$ for 20 min (high eluting time), increased to $98 \%$ B within 5 min, held at $98 \%$ $B$ for another $5 \mathrm{~min}$ for column washing, decreased to $2 \%$ B within $5 \mathrm{~min}$ and held at $2 \% \mathrm{~B}$ for another $10 \mathrm{~min}$ for column balancing. To ensure repeatability of the measurements and stability of the instrument, quality check samples (QC, a mixture of all samples before LC-MS analysis) were analyzed prior to the first sample injection, after every eight injections and at the end of the experiment. Mass spectra were acquired in the positive electrospray ionization mode. For each MS scan, the top five MS (profile mode) scans were followed by MS/MS fragmentation, with the exclusion of charge 1 ions. The collision energy for MS/MS was $35 \mathrm{eV}$. The mass scan range was from 380 to 1800 for MS, and 100 to 1000 for MS/MS.

Each IP extracted plasma sample was analyzed using a nano-LC system (Waters, Milford, MA, USA) coupled with a Thermo linear trap quadrupole (LTQ) Orbitrap Elite mass spectrometer (Orbitrap-MS). For the nano-LCMS analysis, the parameters were the same as those used for in vitro samples, except for the following. A 45-min gradient was applied with a mixture of solvent A and solvent B. The top $10 \mathrm{MS} / \mathrm{MS}$ scans (profile mode) were applied for MS/MS fragmentation.

MaxQuant Data Processing: The acquired LC-MS data was processed by MaxQuant for identification and quantification. All parameters were used in the default settings in MaxQuant, except for database built and the PTM setting. First, human $\alpha$-syn was searched and downloaded as a fasta file format from Uniprot. The file of contaminants was downloaded in csv or Excel format from MaxQuant. The Uniprot ID for $\alpha$ syn is P37840. P37840-2 and P37840-3 refer to isoforms of $\alpha$-syn: for $\mathrm{P} 37840-2$, sequence $103-130$ is missing and for P37840-3, sequence $41-54$ is missing. All items of the contaminant file were copied and pasted into an $\alpha$-syn fasta file, named $\alpha$-syn_with contaminant, as the final database. This strategy sought to reduce the false discovery rate by avoiding a database size that was too small. The complete human protein database is not suggested for reducing the matching time. Contaminants were added to the $\alpha$-syn database to exclude the noise signal from MS that would lead to false positive matches in MaxQuant. For all searches, no fixed PTMs were set. Three kinds of other modifications were manually added in the PTM database. The first was the oxidation of methionine (M) with $15.9949 \mathrm{Da}$ mass addition. The second was nitration in $\mathrm{Y}$ sites with the formula $\mathrm{NO}_{2} \mathrm{H}_{-1}$ and 44.9856 Da mass addition. The third was the following DA derivatives: dopamine-quinone$\mathrm{H}_{2} \mathrm{O}$ (DAQ- $\mathrm{H}_{2} \mathrm{O}$ ) with the formula $\mathrm{C}_{8} \mathrm{H}_{7} \mathrm{NO}$ and $133.0528 \mathrm{Da}$ mass addition; DOPAL- $\mathrm{H}_{2} \mathrm{O}$ modification with the formula $\mathrm{C}_{8} \mathrm{H}_{6} \mathrm{O}_{2}$ and 134.0368 Da mass addition; DAQ with the formula $\mathrm{C}_{8} \mathrm{H}_{9} \mathrm{NO}_{2}$ and 151.0633 Da mass addition; and DC (dopaminochrome) with the formula $\mathrm{C}_{8} \mathrm{H}_{7} \mathrm{NO}_{2}$ and $149.0477 \mathrm{Da}$ mass addition. For in vitro reacted samples, oxidation (M), DAQ- $\mathrm{H}_{2} \mathrm{O}$, and DC- $\mathrm{H}_{2} \mathrm{O}$ were chosen as candidates of variable PTM. Since in vitro samples might have non-covalent bonds with DA derivatives due to the large amount of DA and derivatives in solution, DA derivatives with full mass were also searched for in another experiment, for which DAQ and DC were chosen as candidates of variable PTM. For IP extracted plasma samples, Acetyl-N-terminal, Phosphorylation (STY), Oxidation (M), Glygly (K) (refers to ubiquitination), Nitration $\mathrm{Y}, \mathrm{DAQ}-\mathrm{H}_{2} \mathrm{O}$ and DOPAL- $\mathrm{H}_{2} \mathrm{O}$ modification were chosen as candidates of variable PTM. The txt files exported from MaxQuant were the results of $\alpha$-syn identification.

Quantification of $\alpha$-syn Protein and $\alpha$-syn Modification: The quality of the dataset was checked by performing Principal Components Analysis (PCA). For this, raw data were loaded into Progenesis QI for proteomics (QI) to extract and export all ions with their normalized abundance in each individual pool. Samples (runs) were aligned with non-conflicting peptides, which allowed the comparison of one protein across runs. Ions were filtered and normalized using the QI default parameters to globally adjust all runs to be on the "same scale". Briefly, one run was automatically selected as the normalization reference. Then, for every run, a ratio was taken for the value of the peptide ion abundance in that run to the value in the normalization reference. QI performed a log 10 transformation on all ratio data within each run as well as for all runs, to generate a series of normal distributions. Next, the $\log 10$ ratio distributions were centered by simply adding or subtracting the value required to shift the sample distribution over the normalization reference distribution. To complete the process, a returned "abundance-space ratio" was applied to all values in the sample run being normalized once the scalar had been derived in log space. More detailed information about the default normalization by QI is found at http://www.nonlinear.com/progenesis/qi-forproteomics/v2.0/faq/how-normalisation-works.aspx. The PCA result with tight clustering of QC samples is displayed in Figure S1, Supporting Information. In addition, quantification was done using the identification result of MaxQuant and the normalized intensity exported from QI. In detail, the MaxQuant identification result was exported to derive the LC-MS information ( $\mathrm{m} / \mathrm{z}, \mathrm{RT}, \mathrm{z}$, mass weight) of each peptide ion (unmodified and modified). The matched peptide ions were located from QI exported ion list to prepare a list for all $\alpha$-syn peptides. This was followed by a histogram plot (quantification) complete with error bars using the normalized individual intensities for all matched peptide ions exported from QI. For $\alpha$-syn protein quantification, the average intensity of first three abundant peptides were defined as $\alpha$-syn protein intensity; for modification intensity, the exported intensities were used directly. Histogram plots were made using 
Graphpad Prism 6, using the unpaired parametric $t$-test (with Welch's correction because of the different standard deviation in each group).

Data Availability: The raw data has been submitted to the ProteomeXchange Consortium (http://proteomecentral.proteomexchange.org) via the iProX partner repository with the dataset identifier IPX0002176000.

\section{Supporting Information}

Supporting Information is available from the Wiley Online Library or from the author.

\section{Acknowledgements}

This work is supported by the grants SNF-MOST bilateral grant 156346, MOST 2016YFC1306603, NSFC81573830, and NSFC81774154. The authors thank Professor Joern Dengjel for his help on the use of MaxQuant.

\section{Conflict of Interest}

The authors declare no conflict of interest.

\section{Keywords}

alpha-synuclein, dopamine, Parkinson's disease, plasma

[1] H. A. Lashuel, C. R. Overk, A. Oueslati, E. Masliah, Nat. Rev. Neurosci. 2013, 14, 38 .

[2] B. I. Giasson, I. V. Murray, J. Q. Trojanowski, V. M. Lee, J. Biol. Chem. 2001, 276, 2380.

[3] M. G. Spillantini, M. L. Schmidt, V. M. Y. Lee, J. Q. Trojanowski, R. Jakes, M. Goedert, Nature 1997, 388, 839.

[4] M. Hashimoto, T. Takenouchi, M. Mallory, E. Masliah, A. Takeda, Am. J. Pathol. 2000, 156, 734.

[5] F. E. Herrera, A. Chesi, K. E. Paleologou, A. Schmid, A. Munoz, M. Vendruscolo, S. Gustincich, H. A. Lashuel, P. Carloni, PLoS One 2008, 3, e3394.

[6] T. S. Ulmer, A. Bax, N. B. Cole, R. L. Nussbaum, J. Biol. Chem. 2005, 280, 9595.

[7] H. J. Lee, S. M. Baek, D. H. Ho, J. E. Suk, E. D. Cho, S. J. Lee, Exp. Mol. Med. 2011, 43, 216.

[8] H. Heise, W. Hoyer, S. Becker, O. C. Andronesi, D. Riedel, M. Baldus, Proc. Natl. Acad. Sci. USA 2005, 102, 15871.

[9] J. A. Rodriguez, M. I. Ivanova, M. R. Sawaya, D. Cascio, F. E. Reyes, D. Shi, S. Sangwan, E. L. Guenther, L. M. Johnson, M. Zhang, L. Jiang, M. A. Arbing, B. L. Nannenga, J. Hattne, J. Whitelegge, A. S. Brewster, M. Messerschmidt, B. Boutet, N. K. Sauter, T. Gonen, D. S. Eisenberg, Nature 2015, 525, 486.

[10] J. Lotharius, P. Brundin, Nat. Rev. Neurosci. 2002, 3, 932.

[11] V. Chen, S. Saez-Atienzar, Mov. Disord. 2018, 33, 249.

[12] Y. C. Wong, D. Krainc, Nat. Med. 2017, 23, 1.

[13] J. Xu, S.-Y. Kao, F. J. S. Lee, W. Song, L.-W. Jin, B. A. Yankner, Nat. Med. 2002, 8, 600 .

[14] D. E. Mor, E. Tsika, J. R. Mazzulli, N. S. Gould, H. Kim, M. J. Daniels, S. Doshi, P. Gupta, J. L. Grossman, V. X. Tan, R. G. Kalb, K. A. Caldwell,
G. A. Caldwell, J. H. Wolfe, H. Ischiropoulos, Nat. Neurosci. 2017, 20 , 1560.

[15] S. Roy, Nat. Neurosci. 2017, 20, 1514.

[16] C. L. Pham, S. L. Leong, F. E. Ali, V. B. Kenche, A. F. Hill, S. L. Gras, K. J. Barnham, R. Cappai, J. Mol. Biol. 2009, 387, 771.

[17] M. S. Planchard, S. E. Exley, S. E. Morgan, V. Rangachari, Protein Sci. 2014, 23, 1369.

[18] J. W. Werner-Allen, J. F. DuMond, R. L. Levine, A. Bax, Angew. Chem., Int. Ed. 2016, 55, 7374.

[19] K. S. Kim, J. H. Kang, Bull. Korean Chem. Soc. 2005, 26, 1255.

[20] C. Follmer, E. Coelho-Cerqueira, D. Y. Yatabe-Franco, G. D. T. Araujo, A. S. Pinheiro, G. B. Domont, D. Eliezer, J. Biol. Chem. 2015, 290, 27660.

[21] D. Latawiec, F. Herrera, A. Bek, V. Losasso, M. Candotti, F. Benetti, E. Carlino, A. Kranjc, M. Lazzarino, S. Gustincich, P. Carloni, G. Legname, PLoS One 2010, 5, e9234.

[22] J. R. Mazzulli, M. Armakola, M. Dumoulin, I. Parastatidis, H. Ischiropoulos, J. Biol. Chem. 2007, 282, 31621

[23] S. L. Leong, C. L. Pham, D. Galatis, M. T. Fodero-Tavoletti, K. Perez, A. F. Hill, C. L. Masters, F. E. Ali, K. J. Barnham, R. Cappai, Free Radical Biol. Med. 2009, 46, 1328.

[24] T. Chan, A. M. Chow, X. R. Cheng, D. W. Tang, I. R. Brown, K. Kerman, ACS Chem. Neurosci. 2012, 3, 569.

[25] W. J. Burke, V. B. Kumar, N. Pandey, W. M. Panneton, Q. Gan, M. W. Franko, M. O'Dell, S. W. Li, Y. Pan, H. D. Chung, J. E. Galvin, Acta Neuropathol. 2008, 115, 193.

[26] D. Dibenedetto, G. Rossetti, R. Caliandro, P. Carloni, Biochemistry 2013, 52, 6672 .

[27] S. Corvaglia, B. Sanavio, R. P. Hong Enriquez, B. Sorce, A. Bosco, D. Scaini, S. Sabella, P. P. Pompa, G. Scoles, L. Casalis, Sci. Rep. 2014, 4, 5366.

[28] E. H. Norris, B. I. Giasson, R. Hodara, S. Xu, J. Q. Trojanowski, H. Ischiropoulos, V. M. Lee, J. Biol. Chem. 2005, 280, 21212.

[29] C. L. Pham, R. Cappai, Biosci. Rep. 2013, 33, 807.

[30] C. L. Pham, N. Kirby, K. Wood, T. Ryan, B. Roberts, A. Sokolova, K. J. Barnham, C. L. Masters, R. B. Knott, R. Cappai, C. C. Curtain, A. Rekas, Proteins: Struct., Funct., Bioinf. 2014, 82, 10.

[31] M. Bisaglia, L. Tosatto, F. Munari, I. Tessari, P. P. de Laureto, S. Mammi, L. Bubacco, Biochem. Biophys. Res. Commun. 2010, 394, 424.

[32] A. Oueslati, K. E. Paleologou, B. L. Schneider, P. Aebischer, H. A. Lashuel, J. Neurosci. 2012, 32, 1536.

[33] L. Y. Ma, L. Y. Gao, X. Li, H. Z. Ma, T. Feng, Neurosci. Lett. 2019, 704, 45.

[34] P. Ryan, M. Xu, A. K. Davey, J. J. Danon, G. D. Mellick, M. Kassiou, S. Rudrawar, ACS Chem. Neurosci. 2019, 10, 2209.

[35] R. N. Alcalay, V. Mallett, B. Vanderperre, O. Tavassoly, Y. Dauvilliers, R. Y. J. Wu, J. A. Ruskey, C. S. Leblond, A. Ambalavanan, S. B. Laurent, D. Spiegelman, A. Dionne-Laporte, C. Liong, O. A. Levy, S. Fahn, C. Waters, S. H. Kuo, W. K. Chung, B. Ford, K. S. Marder, U. J. Kang, S. Hassin-Baer, L. Greenbaum, J. F. Trempe, P. Wolf, P. Oliva, X. K. Zhang, L. N. Clark, M. Langlois, P. A. Dion, E. A. Fon, N. Dupre, G. A. Rouleau, Z. Gan-Or, Mov. Disord. 2019, 34, 526.

[36] S. Prasad, P. K. Pal, Mov. Disord. 2018, 33, 403.

[37] M. Colom-Cadena, J. Pegueroles, A. G. Herrmann, C. M. Henstridge, L. Munoz, M. Querol-Vilaseca, C. S. Martin-Paniello, J. LuqueCabecerans, J. Clarimon, O. Belbin, R. Nunez-Llaves, R. Blesa, C. Smith, C. A. McKenzie, M. P. Frosch, A. Roe, J. Fortea, J. Andilla, P. Loza-Alvarez, E. Gelpi, B. T. Hyman, T. L. Spires-Jones, A. Lleo, Brain 2017, 140, 3204.

[38] P. M. Levine, A. Galesic, A. T. Balana, A.-L. Mahul-Mellier, M. X. Navarro, C. A. De Leon, H. A. Lashuel, M. R. Pratt, Proc. Natl. Acad. Sci. USA 2019, 116, 1511.

[39] H. V. Miranda, R. Cassio, L. Correia-Guedes, M. A. Gomes, A. Chegao, E. Miranda, T. Soares, M. Coelho, M. M. Rosa, J. J. Ferreira, T. F. Outeiro, Sci. Rep. 2017, 7, 13713. 
[40] P. G. Foulds, P. Diggle, J. D. Mitchell, A. Parker, M. Hasegawa, M Masuda-Suzukake, D. M. A. Mann, D. Allsop, Sci. Rep. 2013, 3, 2540.

[41] P. G. Foulds, J. D. Mitchell, A. Parker, R. Turner, G. Green, P. Diggle, M. Hasegawa, M. Taylor, D. Mann, D. Allsop, FASEB J. 2011, 25, 4127.

[42] A. W. Schmid, B. Fauvet, M. Moniatte, H. A. Lashuel, Mol. Cell. Proteomics 2013, 12, 3543.

[43] A. Ohrfelt, P. Grognet, N. Andreasen, A. Wallin, E. Vanmechelen, K. Blennow, H. Zetterberg, Neurosci. Lett. 2009, 450, 332.

[44] L. A. Farrelly, R. E. Thompson, S. Zhao, A. E. Lepack, Y. Lyu, N. V. Bhanu, B. Zhang, Y.-H. E. Loh, A. Ramakrishnan, K. C. Vadodaria, K. J. Heard, G. Erikson, T. Nakadai, R. M. Bastle, B. J. Lukasak, H. Zebroski, 3rd, N. A, M. Bader, O. Berton, R. G. Roeder, H. Molina, F. H. Gage, L. Shen, B. A. Garcia, H. Li, T. W. Muir, I. Maze, Nature 2019, 567, 535

[45] I. S. Maze, Alcoholism (NY) 2016, 40, 249 A.

[46] R. E. Whitehead, J. V. Ferrer, J. A. Javitch, J. B. Justice, J. Neurochem. 2001, 76, 1242.

[47] Y. J. Chai, E. Sierecki, V. M. Tomatis, R. S. Gormal, N. Giles, I. C. Morrow, D. Xia, J. Götz, R. G. Parton, B. M. Collins, Y. Gambin, F. A. Meunier, J. Cell Biol. 2016, 214, 705.

[48] Y.-Y. Xie, C.-J. Zhou, Z.-R. Zhou, J. Hong, M.-X. Che, Q.-S. Fu, A.-X. Song, D.-H. Lin, H.-Y. Hu, FASEBJ. 2010, 24, 196.

[49] N. Warren, C. O'Gorman, A. Lehn, D. Siskind, J. Neurol., Neurosurg. Psychiatry 2017, 88, 1060.

[50] P. Belujon, A. A. Grace, Int. J. Neuropsychopharmacol. 2017, 20, 1036.

[51] L. F. Burbulla, P. Song, J. R. Mazzulli, E. Zampese, Y. C. Wong, S. Jeon, D. P. Santos, J. Blanz, C. D. Obermaier, C. Strojny, J. N. Savas, E. Kiskinis, X. Zhuang, R. Kruger, D. J. Surmeier, D. Krainc, Science 2017, 357, 1255

[52] H. Juarez Olguin, D. Calderon Guzman, E. Hernandez Garcia, G. Barragan Mejia, Oxid. Med. Cell. Longevity 2016, 2016, 9730467.

[53] K. A. Conway, J. C. Rochet, R. M. Bieganski, P. T. Lansbury, Science 2001, 294, 1346

[54] S. Hall, Y. Surova, A. Ohrfelt, K. Blennow, H. Zetterberg, O. Hansson, F. S. Swedish Bio, Mov. Disord. 2016, 31, 898.

[55] A. Rekas, R. B. Knott, A. Sokolova, K. J. Barnham, K. A. Perez, C. L. Masters, S. C. Drew, R. Cappai, C. C. Curtain, C. L. Pham, Eur. Biophys. J. 2010, 39, 1407.
[56] E. Ponzini, A. De Palma, L. Cerboni, A. Natalello, R. Rossi, R. Moons, A. Konijnenberg, J. Narkiewicz, G. Legname, F. Sobott, P. Mauri, C. Santambrogio, R. Grandori, J. Biol. Chem. 2019, 294, 5657.

[57] P. Carmo-Goncalves, L. A. do Nascimento, J. R. Cortines, D. Eliezer, L. Romao, C. Follmer, Biochem. Biophys. Res. Commun. 2018, 505, 295.

[58] R. Hummerich, P. Schloss, Neurochem. Int. 2010, 57, 67.

[59] R. Hummerich, V. Costina, P. Findeisen, P. Schloss, ACS Chem. Neurosci. 2015, 6, 1130 .

[60] D. J. Walther, S. Stahlberg, J. Vowinckel, FEBS J. 2011, 278, 4740.

[61] J. Vowinckel, S. Stahlberg, N. Paulmann, K. Bluemlein, M. Grohmann, M. Ralser, D. J. Walther, FEBS Lett. 2012, 586, 3819.

[62] T. S. Lai, C. S. Greenberg, Amino Acidss 2013, 45, 857.

[63] K. B. Johnson, J. M. Thompson, S. W. Watts, Front. Physiol. 2010, 1, 131.

[64] D. J. Walther, J. U. Peter, S. Winter, M. Holtje, N. Paulmann, M. Grohmann, J. Vowinckel, V. Alamo-Bethencourt, C. S. Wilhelm, G. Ahnert-Hilger, M. Bader, Cell 2003, 115, 851.

[65] R. Hummerich, J. O. Thumfart, P. Findeisen, D. Bartsch, P. Schloss, FEBS Lett. 2012, 586, 3421.

[66] S. W. Qiao, J. Piper, G. Haraldsen, I. Oynebraten, B. Fleckenstein, O. Molberg, C. Khosla, L. M. Sollid, J. Immunol. 2005, 174, 1657.

[67] Q. M. Ruan, A. J. Harrington, K. A. Caldwell, G. A. Caldwell, D. G. Standaert, Neurobiol. Dis. 2010, 37, 330.

[68] T. Nakamura, H. Yamashita, Y. Nagano, T. Takahashi, S. Avraham, H. Avraham, M. Matsumoto, S. Nakamura, FEBS Lett. 2002, 521, 190.

[69] T. Takahashi, H. Yamashita, Y. Nagano, T. Nakamura, M. Matsumoto, Mov. Disord. 2006, 21, 1513.

[70] M. M. Hoehn, M. D. Yahr, Onset, Prog. Mortal. 1967, 17, 427.

[71] P. E. Geyer, L. M. Holdt, D. Teupser, M. Mann, Mol. Syst. Biol. 2017, 13, 942.

[72] G. S. Omenn, D. J. States, M. Adamski, T. W. Blackwell, R. Menon, H. Hermjakob, R. Apweiler, B. B. Haab, R. J. Simpson, J. S. Eddes, E. A. Kapp, R. L. Moritz, D. W. Chan, A. J. Rai, A. Admon, R. Aebersold, J. Eng, W. S. Hancock, S. A. Hefta, H. Meyer, Y. K. Paik, J. S. Yoo, P. P. Ping, J. Pounds, J. Adkins, X. H. Qian, R. Wang, V. Wasinger, C. Y. Wu, X. H. Zhao, et al., Proteomics 2005, 5, 3226.

[73] J. Rappsilber, M. Mann, Y. Ishihama, Nat. Protoc. 2007, 2, 1896. 